\title{
Some New Nonlinear Weakly Singular Inequalities and Applications to Volterra-Type Difference Equation
}

\author{
Kelong Zheng, ${ }^{1}$ Wenqiang Feng, ${ }^{2}$ and Chunxiang Guo ${ }^{3}$ \\ ${ }^{1}$ School of Science, Southwest University of Science and Technology, Mianyang, Sichuan 621010, China \\ ${ }^{2}$ Department of Mathematics, University of Tennessee, Knoxville, TN 37996-0612, USA \\ ${ }^{3}$ School of Business, Sichuan University, Chengdu, Sichuan 610064, China
}

Correspondence should be addressed to Chunxiang Guo; guocx70@163.com

Received 3 August 2013; Revised 21 September 2013; Accepted 25 September 2013

Academic Editor: Josef Diblík

Copyright (C) 2013 Kelong Zheng et al. This is an open access article distributed under the Creative Commons Attribution License, which permits unrestricted use, distribution, and reproduction in any medium, provided the original work is properly cited.

Some new nonlinear weakly singular difference inequalities are discussed, which generalize some known weakly singular inequalities and can be used in the analysis of nonlinear Volterra-type difference equations with weakly singular kernel. An application to the upper bound of solutions of a nonlinear difference equation is also presented.

\section{Introduction}

The discrete version of the well-known Gronwall-Bellman inequality is an important tool in the development of the theory of difference equations as well as the analysis of the numerical schemes of differential equations. A great deal of interest has been given to these inequalities, and many results on their generalizations have been found; for example, see [14]. Among them, one of the fundamental cases is Pachpatte's result [3] for the difference inequality:

$$
u(n) \leq a(n)+\sum_{s=0}^{n-1} f(s) u(s)
$$

In particular, due to the study of the behavior and numerical solutions for the singular integral equations, some discrete weakly singular integral inequalities also have drawn more and more attention [5-7]. Dixon and McKee [8] investigated the convergence of discretization methods for the Volterra integral and integrodifferential equations, by using the following inequality:

$$
\begin{array}{r}
x_{i} \leq \psi_{i}+M h^{1-\alpha} \sum_{j=0}^{i-1} \frac{x_{j}}{(i-j)^{\alpha}}, \quad i=1,2, \ldots, N, \\
n>0, N h=T .
\end{array}
$$

Henry [9] presented a linear integral inequality with weakly kernel:

$$
x(t) \leq a(t)+\int_{0}^{t}(t-s)^{\beta-1} b(s) x(s) d s
$$

to investigate some qualitative properties for a parabolic equation. The corresponding discrete version was discussed by Slodička [10]. But he studied the case $\tau_{k}=\tau$, that is, the case of constant differences. Furthermore, the first formulation of the inequality with a nonlinearity and $\tau_{k}$ nonconstant was studied in [6], in which the general nonlinear discrete case as follows:

$$
x_{n} \leq a_{n}+\sum_{k=0}^{n-1}\left(t_{n}-t_{k}\right)^{\beta-1} \tau_{k} b_{k} \omega\left(x_{k}\right)
$$

was considered. However, his results are based on the so-called "( $q)$ condition": (1) $\omega$ satisfies $e^{-q t}[\omega(u)]^{q} \leq$ $R(t) \omega\left(e^{-q t}\right) u^{q} ;$ (2) there exists $c>0$ such that $a_{n} e^{-\tau t_{n}} \leq c$. Recently, a new nonlinear difference inequality:

$$
x_{n}^{\alpha} \leq a_{n}+\sum_{k=0}^{n-1}\left(t_{n}-t_{k}\right)^{\beta-1} \tau_{k} b_{k} x_{k}^{\lambda}
$$

was discussed by Yang et al. [11]. For other new weakly singular inequalities, lots of work can be found, for example, in [12-22] and references therein. 
In this paper, we investigate the new nonlinear weakly singular inequality:

$$
x_{n} \leq a_{n}+\sum_{k=0}^{n-1}\left(t_{n}-t_{k}\right)^{\beta-1} \tau_{k} b_{k} \omega\left(x_{k}\right)
$$

where $0<\beta \leq 1, t_{0}=0, \tau_{k}=t_{k+1}-t_{k}$, $\sup _{k \in \mathbb{N}} \tau_{k}=\tau$, and $\lim _{t \rightarrow \infty} t_{k}=\infty$. Compared to the existing result, our result does not need the so-called " $(q)$ condition" proposed in [6] and can be used to obtain pointwise explicit bounds on solutions for a class of more general weakly singular inequalities of Volterra type. Finally, we also present an application to Volterra-type difference equation with weakly singular kernel.

\section{Preliminaries}

Let $\mathbb{R}$ be the set of real numbers, $\mathbb{R}_{+}=(0, \infty)$, and $\mathbb{N}=$ $\{0,1,2, \ldots\} . C(X, Y)$ denotes the collection of continuous functions from the set $X$ to the set $Y$. As usual, the empty sum is taken to be 0 .

Lemma 1 (Discrete Jensen inequality, [11]). Let $A_{1}, A_{2}, \ldots$, $A_{n}$ be nonnegative real numbers, and let $r>1$ be a real number. Then,

$$
\left(A_{1}+A_{2}+\cdots+A_{n}\right)^{r} \leq n^{r-1}\left(A_{1}^{r}+A_{2}^{r}+\cdots+A_{n}^{r}\right) .
$$

Lemma 2 (Discrete Hölder inequality, [11]). Let $a_{i}, b_{i}(i=$ $1,2, \ldots, n)$ be nonnegative real numbers, and let $p, q$ be positive numbers such that $(1 / p)+(1 / q)=1(\operatorname{or} p=1, q=\infty)$. Then,

$$
\sum_{i=1}^{n} a_{i} b_{i} \leq\left(\sum_{i=1}^{n} a_{i}^{p}\right)^{1 / p}\left(\sum_{i=1}^{n} b_{i}^{q}\right)^{1 / q}
$$

Furthermore, take $p=q=2$; then, one gets the discrete Cauchy-Schwarz inequality.

Lemma 3. Suppose that $\omega(u) \in C\left(\mathbb{R}_{+}, \mathbb{R}_{+}\right)$is nondecreasing. Let $a_{n}, c_{n}$ be nonnegative and nondecreasing in $n$. If $y_{n}$ is nonnegative such that

$$
y_{n} \leq a_{n}+c_{n} \sum_{k=0}^{n-1} b_{k} \omega\left(y_{k}\right), \quad n \in \mathbb{N} \text {. }
$$

Then,

$$
y_{n} \leq \Omega^{-1}\left[\Omega\left(a_{n}\right)+c_{n} \sum_{k=0}^{n-1} b_{k}\right], \quad 0 \leq n \leq M,
$$

where $\Omega(v)=\int_{v_{0}}^{v}(1 / \omega(s)) d s, v \geq v_{0}, \Omega^{-1}$ is the inverse function of $\Omega$, and $M$ is defined by

$$
M=\sup \left\{i: \Omega\left(a_{i}\right)+c_{i} \sum_{k=0}^{i-1} b_{k} \in \operatorname{Dom}\left(\Omega^{-1}\right)\right\} .
$$

\section{Main Results}

Assume that

$\left(A_{1}\right) a_{n}, b_{n}$ are nonnegative functions for $n \in \mathbb{N}$, respectively;

$\left(A_{2}\right) \omega(u) \in C\left(\mathbb{R}_{+}, \mathbb{R}_{+}\right)$is nondecreasing and $\omega(0)=$ 0 .

Define $\tilde{a}_{n}=\max _{0 \leq k \leq n, k \in \mathbb{N}} a_{k}$ and $\tau=\max _{0 \leq k \leq n-1, k \in \mathbb{N}} \tau_{k}$, where $\tau_{k}$ is the variable time step.

Theorem 4. Under assumptions $\left(A_{1}\right)$ and $\left(A_{2}\right)$, if $x_{n}$ is nonnegative such that (6), then

(1) for $0<\beta \leq 1 / 2$, letting $p=1+\beta$ and $q=(1+\beta) / \beta$, one has

$$
\begin{aligned}
x_{n} \leq\left[\Omega^{-1}(\Omega\right. & \left(2^{q-1} \widetilde{a}_{n}^{q}\right)+2^{q-1} \tau^{1-(q / p) \beta^{2}} \\
& \left.\left.\times K^{q / p}(\beta) e^{q \tau t_{n}} \sum_{k=0}^{n-1} e^{-q \tau t_{k}} b_{k}^{q}\right)\right]^{1 / q},
\end{aligned}
$$

for $0 \leq n \leq N_{1}$, where $\Omega(u)=\int_{u_{0}}^{u}\left(1 / \omega^{q}\left(s^{1 / q}\right)\right) d s, u \geq$ $u_{0} \geq 0, \Omega^{-1}$ is the inverse function of $\Omega$,

$$
K(\beta)=(1+\beta)^{-\beta^{2}} \Gamma\left(\beta^{2}\right),
$$

and $N_{1}$ is the largest integer number such that

$$
\begin{aligned}
& \Omega\left(2^{q-1} \widetilde{a}_{n}^{q}\right)+2^{q-1} \tau^{1-(q / p) \beta^{2}} K^{q / p}(\beta) e^{q \tau t_{n}} \\
& \times \sum_{k=0}^{n-1} e^{-q \tau t_{k}} b_{k}^{q} \in \operatorname{Dom}\left(\Omega^{-1}\right) ;
\end{aligned}
$$

(2) for $1 / 2<\beta \leq 1$, letting $p=2$ and $q=2$, one has

$$
x_{n} \leq\left[\Omega^{-1}\left(\Omega\left(2 \widetilde{a}_{n}^{2}\right)+B(\beta) \tau^{2-2 \beta} e^{2 \tau t_{n}} \sum_{k=0}^{n-1} e^{-2 \tau t_{k}} b_{k}^{2}\right)\right]^{1 / 2},
$$

for $0 \leq n \leq N_{2}$, where $\Omega(u)=\int_{u_{0}}^{u}\left(1 / \omega^{2}\left(s^{1 / 2}\right)\right) d s, u \geq$ $u_{0} \geq 0$,

$$
B(\beta)=4^{1-\beta} \Gamma(2 \beta-1), \quad \beta>\frac{1}{2},
$$

and $N_{2}$ is the largest integer number such that

$$
\Omega\left(2 \widetilde{a}_{n}^{2}\right)+B(\beta) \tau^{2-2 \beta} e^{2 \tau t_{n}} \sum_{k=0}^{n-1} e^{-2 \tau t_{k}} b_{k}^{2} \in \operatorname{Dom}\left(\Omega^{-1}\right) .
$$

Proof. By definition of $\tilde{a}_{n}$ and assumption $\left(A_{1}\right), \widetilde{a}_{n}$ is nonnegative and nondecreasing and $\widetilde{a}_{n} \geq a_{n}$. It follows from (6) that

$$
x_{n} \leq \tilde{a}_{n}+\sum_{k=0}^{n-1}\left(t_{n}-t_{k}\right)^{\beta-1} \tau_{k} b_{k} \omega\left(x_{k}\right) \text {. }
$$


(1) If $0<\beta \leq 1 / 2$, using Lemma 2 with the indices $p=$ $1+\beta, q=(1+\beta) / \beta$ for $(18)$, we get

$$
\begin{aligned}
x_{n} \leq & \tilde{a}_{n}+\sum_{k=0}^{n-1}\left(t_{n}-t_{k}\right)^{\beta-1} \tau_{k}^{1 / p} \tau_{k}^{1 / q} e^{\tau t_{k}} e^{-\tau t_{k}} b_{k} \omega\left(x_{k}\right) \\
\leq & \tilde{a}_{n}+\tau^{1 / q} \sum_{k=0}^{n-1}\left(t_{n}-t_{k}\right)^{\beta-1} \tau_{k}^{1 / p} e^{\tau t_{k}} e^{-\tau t_{k}} b_{k} \omega\left(x_{k}\right) \\
\leq & \tilde{a}_{n}+\tau^{1 / q}\left[\sum_{k=0}^{n-1}\left(t_{n}-t_{k}\right)^{p(\beta-1)} \tau_{k} e^{p \tau t_{k}}\right]^{1 / p} \\
& \times\left[\sum_{k=0}^{n-1} e^{-q \tau t_{k}} b_{k}^{q} \omega^{q}\left(x_{k}\right)\right]^{1 / q} .
\end{aligned}
$$

By Lemma 1, the inequality above yields

$$
\begin{aligned}
x_{n}^{q} \leq & 2^{q-1} \widetilde{a}_{n}^{q}+2^{q-1} \tau\left[\sum_{k=0}^{n-1}\left(t_{n}-t_{k}\right)^{p(\beta-1)} \tau_{k} e^{p \tau t_{k}}\right]^{q / p} \\
& \times\left[\sum_{k=0}^{n-1} e^{-q \tau t_{k}} b_{k}^{q} \omega^{q}\left(x_{k}\right)\right] .
\end{aligned}
$$

Consider that

$$
\begin{aligned}
& \sum_{k=0}^{n-1}\left(t_{n}-t_{k}\right)^{p(\beta-1)} \tau_{k} e^{p \tau t_{k}} \\
& \quad \leq \int_{0}^{t_{n}}\left(t_{n}-s\right)^{p(\beta-1)} e^{p \tau s} d s \\
& \quad=e^{p \tau t_{n}} \int_{0}^{t_{n}} \eta^{p(\beta-1)} e^{-p \tau \eta} d \eta, \\
& \quad=\frac{e^{p \tau t_{n}}}{(p \tau)^{1+p(\beta-1)}} \int_{0}^{p \tau t_{n}} \sigma^{p(\beta-1) e^{-\sigma}} d \sigma \leq K(\beta) \tau^{-\beta^{2}} e^{p \tau t_{n}},
\end{aligned}
$$

where $K(\beta)=(1+\beta)^{-\beta^{2}} \Gamma\left(\beta^{2}\right)$ and $\Gamma(z)=\int_{0}^{\infty} u^{z-1}$ $e^{-u} d u,(\operatorname{Re} z>0)$ is the well-known $G$-function. Thus, we have

$$
\begin{aligned}
x_{n}^{q} \leq & 2^{q-1} \widetilde{a}_{n}^{q}+2^{q-1} \tau^{1-(q / p) \beta^{2}} \\
& \times K^{q / p}(\beta) e^{q \tau t_{n}} \sum_{k=0}^{n-1} e^{-q \tau t_{k}} b_{k}^{q} \omega^{q}\left(x_{k}\right) .
\end{aligned}
$$

Let $v_{n}=x_{n}^{q}, A_{n}=2^{q-1} \widetilde{a}_{n}^{q}$, and $C_{n}=2^{q-1} \tau^{1-(q / p) \beta^{2}}$ $K^{q / p}(\beta) e^{q \tau t_{n}}$. Obviously, $A_{n}, C_{n}$ are nondecreasing for $n \in \mathbb{N}$ and $\omega^{q}\left(v_{k}^{1 / q}\right)$ satisfies the assumption $\left(A_{2}\right)$. Equation (22) can be rewritten as

$$
v_{n} \leq A_{n}+C_{n} \sum_{k=0}^{n-1} e^{-q \tau t_{k}} b_{k}^{q} \omega^{q}\left(v_{k}^{1 / q}\right)
$$

which is similar to inequality (9). Using Lemma 3, from (23), we have

$$
v_{n} \leq \Omega^{-1}\left[\left(\Omega\left(A_{n}\right)+C_{n} \sum_{k=0}^{n-1} e^{-q \tau t_{k}} b_{k}^{q}\right)\right],
$$

for $0 \leq n \leq N_{1}$, where $N_{1}$ is the largest integer number such that

$$
\Omega\left(A_{n}\right)+C_{n} \sum_{k=0}^{n-1} e^{-q \tau t_{k}} b_{k}^{q} \in \operatorname{Dom}\left(\Omega^{-1}\right) .
$$

Therefore, by $x_{n}=v_{n}^{1 / q}$, (12) holds for $0 \leq n \leq N_{1}$.

(2) If $1 / 2<\beta \leq 1$, applying Cauchy-Schwarz inequality for (18), that is, $p=q=2$, we get

$$
\begin{aligned}
x_{n} \leq & \widetilde{a}_{n}+\sum_{k=0}^{n-1}\left(t_{n}-t_{k}\right)^{\beta-1} \tau_{k}^{1 / 2} \tau_{k}^{1 / 2} e^{\tau t_{k}} e^{-\tau t_{k}} b_{k} \omega\left(x_{k}\right) \\
\leq & \tilde{a}_{n}+\tau^{1 / 2} \sum_{k=0}^{n-1}\left(t_{n}-t_{k}\right)^{\beta-1} \tau_{k}^{1 / 2} e^{\tau t_{k}} e^{-\tau t_{k}} b_{k} \omega\left(x_{k}\right) \\
\leq & \tilde{a}_{n}+\tau^{1 / 2}\left[\sum_{k=0}^{n-1}\left(t_{n}-t_{k}\right)^{2(\beta-1)} \tau_{k} e^{2 \tau t_{k}}\right]^{1 / 2} \\
& \times\left[\sum_{k=0}^{n-1} e^{-2 \tau t_{k}} b_{k}^{2} \omega^{2}\left(x_{k}\right)\right]^{1 / 2} .
\end{aligned}
$$

By Lemma 1, the inequality above yields

$$
\begin{aligned}
x_{n}^{2} \leq & 2 \widetilde{a}_{n}^{2}+2 \tau\left[\sum_{k=0}^{n-1}\left(t_{n}-t_{k}\right)^{2(\beta-1)} \tau_{k} e^{2 \tau t_{k}}\right] \\
& \times\left[\sum_{k=0}^{n-1} e^{-2 \tau t_{k}} b_{k}^{2} \omega^{2}\left(x_{k}\right)\right] .
\end{aligned}
$$

Because

$$
\begin{aligned}
& \sum_{k=0}^{n-1}\left(t_{n}-t_{k}\right)^{2(\beta-1)} \tau_{k} e^{2 \tau t_{k}} \\
& \quad \leq \int_{0}^{t_{n}}\left(t_{n}-s\right)^{2(\beta-1)} e^{2 \tau s} d s \\
& \quad=\frac{e^{2 \tau t_{n}}}{(2 \tau)^{2 \beta-1}} \int_{0}^{2 \tau t_{n}} \sigma^{2(\beta-1) e^{-\sigma}} d \sigma \\
& \quad \leq \frac{1}{2} B(\beta) \tau^{1-2 \beta} e^{2 \tau t_{n}},
\end{aligned}
$$

where $B(\beta)=4^{1-\beta} \Gamma(2 \beta-1), \beta>1 / 2$, it follows from (27) that

$$
x_{n}^{2} \leq 2 \widetilde{a}_{n}^{2}+B(\beta) \tau^{2-2 \beta} e^{2 \tau t_{n}}\left[\sum_{k=0}^{n-1} e^{-2 \tau t_{k}} b_{k}^{2} \omega^{2}\left(x_{k}\right)\right] .
$$


Let $v_{n}=x_{n}^{2}, A_{n}=2 \widetilde{a}_{n}^{2}$, and $C_{n}=B(\beta) \tau^{2-2 \beta} e^{2 \tau t_{n}}$. Similarly, $A_{n}, C_{n}$ also are nondecreasing for $n \in$ $\mathbb{N}$ and $\omega^{2}\left(v_{k}^{1 / 2}\right)$ also satisfies the assumption $\left(A_{2}\right)$. Equation (29) can be rewritten as

$$
v_{n} \leq A_{n}+C_{n}\left(\sum_{k=0}^{n-1} e^{-2 \tau t_{k}} b_{k}^{2} \omega^{2}\left(v_{k}^{1 / 2}\right)\right),
$$

which also is similar to inequality (9). Using Lemma 3, from (30), we have

$$
v_{n} \leq\left[\Omega^{-1}\left(\Omega\left(A_{n}\right)+C_{n} \sum_{k=0}^{n-1} e^{-2 \tau t_{k}} b_{k}^{2}\right)\right],
$$

for $0 \leq n \leq N_{2}$, and $N_{2}$ is the largest integer number such that

$$
\Omega\left(A_{n}\right)+C_{n} \sum_{k=0}^{n-1} e^{-2 \tau t_{k}} b_{k}^{2} \in \operatorname{Dom}\left(\Omega^{-1}\right) .
$$

Clearly, by $x_{n}=v_{n}^{1 / 2},(15)$ also holds for $0 \leq n \leq N_{2}$.

Remark 5. Here, we note that the most significant work in the study of weakly singular inequalities is Medved's method, originally presented in the paper [6] and also applied in the paper [18]. But his result holds under the assumption " $\omega(u)$ satisfies the condition (q)," that is, " $e^{-q t}[\omega(u)]^{q} \leq$ $R(t) \omega\left(e^{-q t} u^{q}\right)$, where $R(t)$ is a continuous, nonnegative function." In our result, the condition $(q)$ is eliminated.

Corollary 6. Under assumptions $\left(A_{1}\right)$ and $\left(A_{2}\right)$, let $v>0$, $\mu>0(\nu>\mu)$. If $x_{n}$ is nonnegative such that

$$
x_{n}^{\nu} \leq a_{n}+\sum_{k=0}^{n-1}\left(t_{n}-t_{k}\right)^{\beta-1} \tau_{k} b_{k} x_{k}^{\mu}
$$

then

(1) if $0<\beta \leq 1 / 2$, let $p=1+\beta$ and $q=(1+\beta) / \beta$, and one gets

$$
\begin{aligned}
x_{n} \leq[ & \left(2^{q-1} \widetilde{a}_{n}^{q}\right)^{(\nu-\mu) / \nu}+\frac{\nu-\mu}{\nu} 2^{q-1} \tau^{1-(q / p) \beta^{2}} \\
& \left.\times K^{q / p}(\beta) e^{q \tau t_{n}} \sum_{k=0}^{n-1} e^{-q \tau t_{k}} b_{k}^{q}\right]^{1 /(\nu-\mu) q}
\end{aligned}
$$

for $n \geq 0$, where $K(\beta)$ is defined as in Theorem 4;

(2) if $1 / 2<\beta \leq 1$, let $p=q=2$, and one gets

$$
\begin{gathered}
x_{n} \leq\left[\left(2 \widetilde{a}_{n}^{2}\right)^{(\nu-\mu) / \nu}+\frac{\nu-\mu}{\nu} B(\beta) \tau^{2-2 \beta}\right. \\
\left.\times e^{2 \tau t_{n}} \sum_{k=0}^{n-1} e^{-2 \tau t_{k}} b_{k}^{2}\right]^{1 / 2(\nu-\mu)},
\end{gathered}
$$

Proof. Let $z_{n}=x_{n}^{\nu}$, then $x_{n}=z_{n}^{1 / \nu}$ and $x_{n}^{\mu}=z_{n}^{\mu / \nu}$. From (33), we have

$$
z_{n} \leq a_{n}+\sum_{k=0}^{n-1}\left(t_{n}-t_{k}\right)^{\beta-1} \tau_{k} b_{k} z_{k}^{\mu / \nu}
$$

Clearly, $\omega\left(z_{k}\right)=z_{k}^{\mu / \nu}$ satisfies the assumption $\left(A_{2}\right)$. According to the definition of $\Omega$ in Theorem 4 , for $0<\beta \leq 1 / 2$, letting $u_{0}=0$, we have

$$
\begin{aligned}
& \Omega(u)=\int_{u_{0}}^{u} \frac{1}{\omega^{q}\left(s^{1 / q}\right)} d s=\int_{0}^{u} \frac{d s}{s^{\mu / v}}=\frac{v}{v-\mu} u^{(\nu-\mu) / \nu}, \\
& \Omega^{-1}(u)=\left(\frac{v-\mu}{v} u\right)^{\nu /(\nu-\mu)}, \quad \operatorname{Dom}\left(\Omega^{-1}\right)=[0, \infty) .
\end{aligned}
$$

It can be seen easily from (38) that $N_{1}=\infty$. Substituting (37) and (38) into (12), we get

$$
\begin{aligned}
z_{n} \leq[ & \left(2^{q-1} \widetilde{a}_{n}^{q}\right)^{(\nu-\mu) / \nu}+\frac{\nu-\mu}{\nu} 2^{q-1} \tau^{1-(q / p) \beta^{2}} \\
& \left.\times K^{q / p}(\beta) e^{q \tau t_{n}} \sum_{k=0}^{n-1} e^{-q \tau t_{k}} b_{k}^{q}\right]^{\nu /(\nu-\mu) q} .
\end{aligned}
$$

In view of $x_{n}=z_{n}^{1 / \nu}$, we can obtain (34). For the case that $1 / 2<\beta \leq 1$, in fact, $\Omega$ and $\Omega^{-1}$ are the same as (37) and (38), respectively. So, it follows from (37), (38), and (15) that

$$
\begin{gathered}
x_{n} \leq\left[\left(2 \widetilde{a}_{n}^{2}\right)^{(\nu-\mu) / \nu}+\frac{\nu-\mu}{\nu} B(\beta) \tau^{2-2 \beta}\right. \\
\left.\quad \times e^{2 \tau t_{n}} \sum_{k=0}^{n-1} e^{-2 \tau t_{k}} b_{k}^{2}\right]^{1 / 2(\nu-\mu)}
\end{gathered}
$$

for $n>0$.

Remark 7. In [11], Yang et al. investigated inequality (33), under the assumption that $a_{n}$ is nondecreasing. Clearly, our result does not need such condition, and we get a more concise formula.

Remark 8. Letting $v=2$ and $\mu=1$, we can get the interesting Henry version of the Ou-Iang-Pachpatte-type difference inequality [3]. Thus, our result is a more general discrete analogue for such inequality.

Corollary 9. Under assumptions $\left(A_{1}\right)$ and $\left(A_{2}\right)$, if $x_{n}$ is nonnegative such that

$$
x_{n} \leq a_{n}+\sum_{k=0}^{n-1}\left(t_{n}-t_{k}\right)^{\beta-1} \tau_{k} b_{k} x_{k}
$$


then

(1) if $0<\beta \leq 1 / 2$, let $p=1+\beta$ and $q=(1+\beta) / \beta$, and one gets

$$
\begin{gathered}
x_{n} \leq 2^{(q-1) / q} \widetilde{a}_{n} \exp \left(2^{(q-1) / q} \tau^{1-(q / p) \beta^{2}} K^{q / p}(\beta)\right. \\
\left.\times e^{q \tau t_{n}} \sum_{k=0}^{n-1} e^{-q \tau t_{k}} b_{k}^{q}\right),
\end{gathered}
$$

for $n \geq 0$, where $K(\beta)$ is defined as in Theorem 4;

(2) if $1 / 2<\beta \leq 1$, let $p=q=2$, and one gets

$$
x_{n} \leq \sqrt{2} \widetilde{a}_{n} \exp \left(\frac{1}{2} B(\beta) \tau^{2-2 \beta} e^{2 \tau t_{n}} \sum_{k=0}^{n-1} e^{-2 \tau t_{k}} b_{k}^{2}\right) \text {, }
$$

for $n \geq 0$, where $B(\beta)$ is defined as in Theorem 4 .

Proof. In (41), $\omega(u)=u$ also satisfies the assumption $\left(A_{2}\right)$. Thus, we have

$$
\begin{gathered}
\Omega(u)=\int_{u_{0}}^{u} \frac{d s}{s}=\ln \frac{u}{u_{0}}, \quad \Omega^{-1}(u)=u_{0} \exp (u), \\
\operatorname{Dom}\left(\Omega^{-1}\right)=[0, \infty) .
\end{gathered}
$$

Similarly to the computation in Corollary 6, the estimates (42) and (43) hold, respectively.

\section{Application}

In this section, we apply our results to discuss the upper bound of solution of a Volterra type difference equation with weakly singular kernel.

Consider the following the inequality:

$$
x_{n} \leq 1+\sum_{k=0}^{n-1}\left(t_{n}-t_{k}\right)^{-1 / 2} \tau_{k} \sqrt{x_{k}} .
$$

Obviously, (45) is the special case of inequality (6), then we get

$$
a_{n}=1, \quad \beta=\frac{1}{2}, \quad \omega=\sqrt{u} \text {. }
$$

Thus, we can take $p=1+\beta=3 / 2$ and $q=(1+\beta) / \beta=3$; then, $q / p=2$. Moreover,

$$
\begin{gathered}
\tilde{a}_{n}=1, \\
K(\beta)=(1+\beta)^{-\beta^{2}} \Gamma\left(\beta^{2}\right)=\left(\frac{3}{2}\right)^{-1 / 4} \Gamma\left(\frac{1}{4}\right), \\
\Omega(u)=\int_{0}^{u} \frac{d s}{\sqrt{s}}=2 \sqrt{u}, \quad \Omega^{-1}(u)=\frac{u^{2}}{4} .
\end{gathered}
$$

According to Theorem 4, we obtain

$$
\begin{aligned}
x_{n} \leq & {\left[\Omega^{-1}\left(\Omega\left(2^{q-1} \widetilde{a}_{n}^{q}\right)+2^{q-1} \tau^{1-(q / p) \beta^{2}}\right)\right.} \\
& \left.\times K^{q / p}(\beta) e^{q \tau t_{n}} \sum_{k=0}^{n-1} e^{-q \tau t_{k}} b_{k}^{q}\right]^{1 / q} \\
= & {\left[\Omega^{-1}\left(\Omega(4)+4 \tau^{1 / 2}\left(\frac{3}{2}\right)^{-1 / 2}\right)\right.} \\
= & \left.\times \Gamma^{2}\left(\frac{1}{4}\right) e^{3 \tau t_{n}} \sum_{k=0}^{n-1} e^{-3 \tau t_{k}} b_{k}^{3}\right]^{1 / 3}\left(4+\frac{4}{3} \sqrt{6} \tau^{1 / 2} \Gamma^{2}\left(\frac{1}{4}\right) e^{3 \tau t_{n}} \sum_{k=0}^{n-1} e^{-3 \tau t_{k}} b_{k}^{3}\right]^{1 / 3} \\
= & 4^{-1 / 3}\left(4+\frac{4}{3} \sqrt{6} \tau^{1 / 2} \Gamma^{2}\left(\frac{1}{4}\right) e^{3 \tau t_{n}} \sum_{k=0}^{n-1} e^{-3 \tau t_{k}} b_{k}^{3}\right)^{2 / 3}
\end{aligned}
$$

for $n>0$, which indicates that we get the upper bound of $x_{n}$.

\section{Conflict of Interests}

The authors declare that there is no conflict of interests regarding the publication of this paper.

\section{Acknowledgments}

This work is supported by the Doctoral Program Research Funds of Southwest University of Science and Technology (no. 11zx7129) and the Fundamental Research Funds for the Central Universities (no. skqy201324). The work is also supported by the Applied Basic Research Project of Sichuan Province (no. 2013JY0096).

\section{References}

[1] R. P. Agarwal, S. Deng, and W. Zhang, "Generalization of a retarded Gronwall-like inequality and its applications," Applied Mathematics and Computation, vol. 165, no. 3, pp. 599-612, 2005.

[2] S. Deng, "Nonlinear discrete inequalities with two variables and their applications," Applied Mathematics and Computation, vol. 217, no. 5, pp. 2217-2225, 2010.

[3] B. G. Pachpatte, "On some fundamental integral inequalities and their discrete analogues," Journal of Inequalities in Pure and Applied Mathematics, vol. 2, no. 2, article 15, 2001.

[4] Y. Wu, X. Li, and S. Deng, "Nonlinear delay discrete inequalities and their applications to Volterra type difference equations," Advances in Difference Equations, vol. 2010, Article ID 795145, 14 pages, 2010.

[5] M. Medved, "A new approach to an analysis of Henry type integral inequalities and their Bihari type versions," Journal of Mathematical Analysis and Applications, vol. 214, no. 2, pp. 349366, 1997. 
[6] M. Medved, "Nonlinear singular difference inequalities suitable for discretizations of parabolic equations," Demonstratio Mathematica, vol. 33, no. 3, pp. 517-525, 2000.

[7] K. Zheng, H. Wang, and C. Guo, "On nonlinear discrete weakly singular inequalities and applications to Volterra-type difference equations," Advances in Difference Equations, vol. 2013, article 239, 2013.

[8] J. Dixon and S. McKee, "Weakly singular discrete Gronwall inequalities," Zeitschrift für Angewandte Mathematik und Mechanik, vol. 66, no. 11, pp. 535-544, 1986.

[9] D. Henry, Geometric Theory of Semilinear Parabolic Equations, vol. 840 of Lecture Notes in Mathematics, Springer, New York, NY, USA, 1981.

[10] M. Slodička, "Semigroup formulation of Rothe's method: application to parabolic problems," Commentationes Mathematicae Universitatis Carolinae, vol. 33, no. 2, pp. 245-260, 1992.

[11] E.-H. Yang, Q.-H. Ma, and M.-C. Tan, "Discrete analogues of a new class of nonlinear Volterra singular integral inequalities," Journal of Jinan University, vol. 28, no. 1, pp. 1-6, 2007.

[12] W.-S. Cheung, Q.-H. Ma, and S. Tseng, "Some new nonlinear weakly singular integral inequalities of Wendroff type with applications," Journal of Inequalities and Applications, vol. 2008, Article ID 909156, 12 pages, 2008.

[13] S. Deng and C. Prather, "Generalization of an impulsive nonlinear singular Gronwall-Bihari inequality with delay," Journal of Inequalities in Pure and Applied Mathematics, vol. 9, no. 2, article 34, 2008.

[14] F. Lakhal, "A new nonlinear integral inequality of Wendroff type with continuous and weakly singular kernel and its application," Journal of Mathematical Inequalities, vol. 6, no. 3, pp. 367-379, 2012.

[15] Q. H. Ma and E. H. Yang, "Estimates on solutions of some weakly singular Volterra integral inequalities," Acta Mathematicae Applicatae Sinica, vol. 25, no. 3, pp. 505-515, 2002.

[16] Q. H. Ma and J. Pečarić, "Some new explicit bounds for weakly singular integral inequalities with applications to fractional differential and integral equations," Journal of Mathematical Analysis and Applications, vol. 341, no. 2, pp. 894-905, 2008.

[17] S. Mazouzi and N.-E. Tatar, "New bounds for solutions of a singular integro-differential inequality," Mathematical Inequalities \& Applications, vol. 13, no. 2, pp. 427-435, 2010.

[18] M. Medved, "On singular versions of Bihari and WendroffPachpatte type integral inequalities and their application," Tatra Mountains Mathematical Publications, vol. 38, pp. 163-174, 2007.

[19] S. Szufla, "On the Volterra integral equation with weakly singular kernel," Mathematica Bohemica, vol. 131, no. 3, pp. 225231, 2006.

[20] N.-E. Tatar, "On an integral inequality with a kernel singular in time and space," Journal of Inequalities in Pure and Applied Mathematics, vol. 4, no. 4, article 82, 2003.

[21] H. Wang and K. Zheng, "Some nonlinear weakly singular integral inequalities with two variables and applications," Journal of Inequalities and Applications, vol. 2010, Article ID 345701, 12 pages, 2010.

[22] K. Zheng, "Bounds on some new weakly singular Wendrofftype integral inequalities and applications," Journal of Inequalities and Applications, vol. 2013, article 159, 2013. 


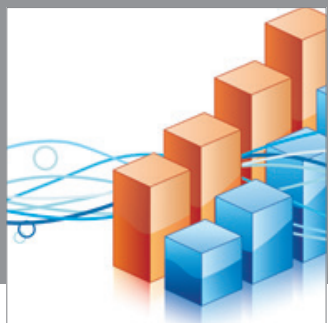

Advances in

Operations Research

mansans

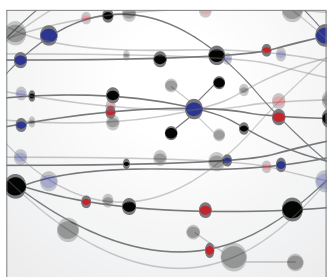

The Scientific World Journal
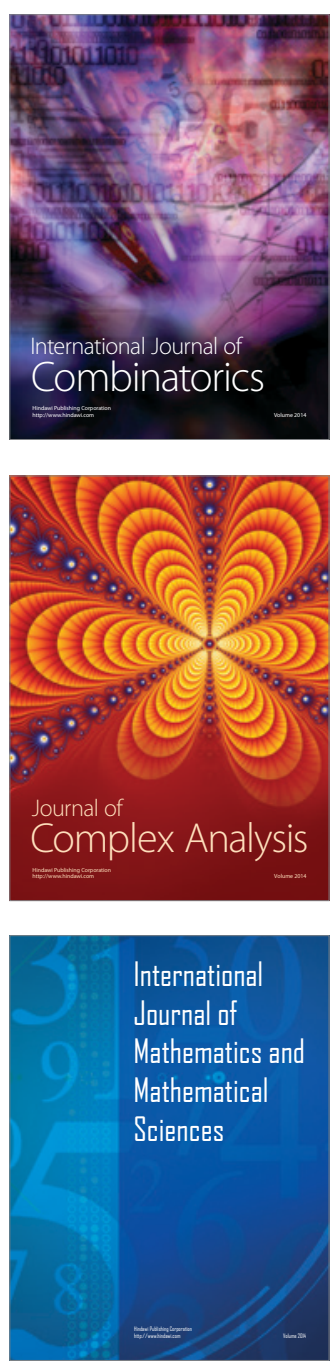
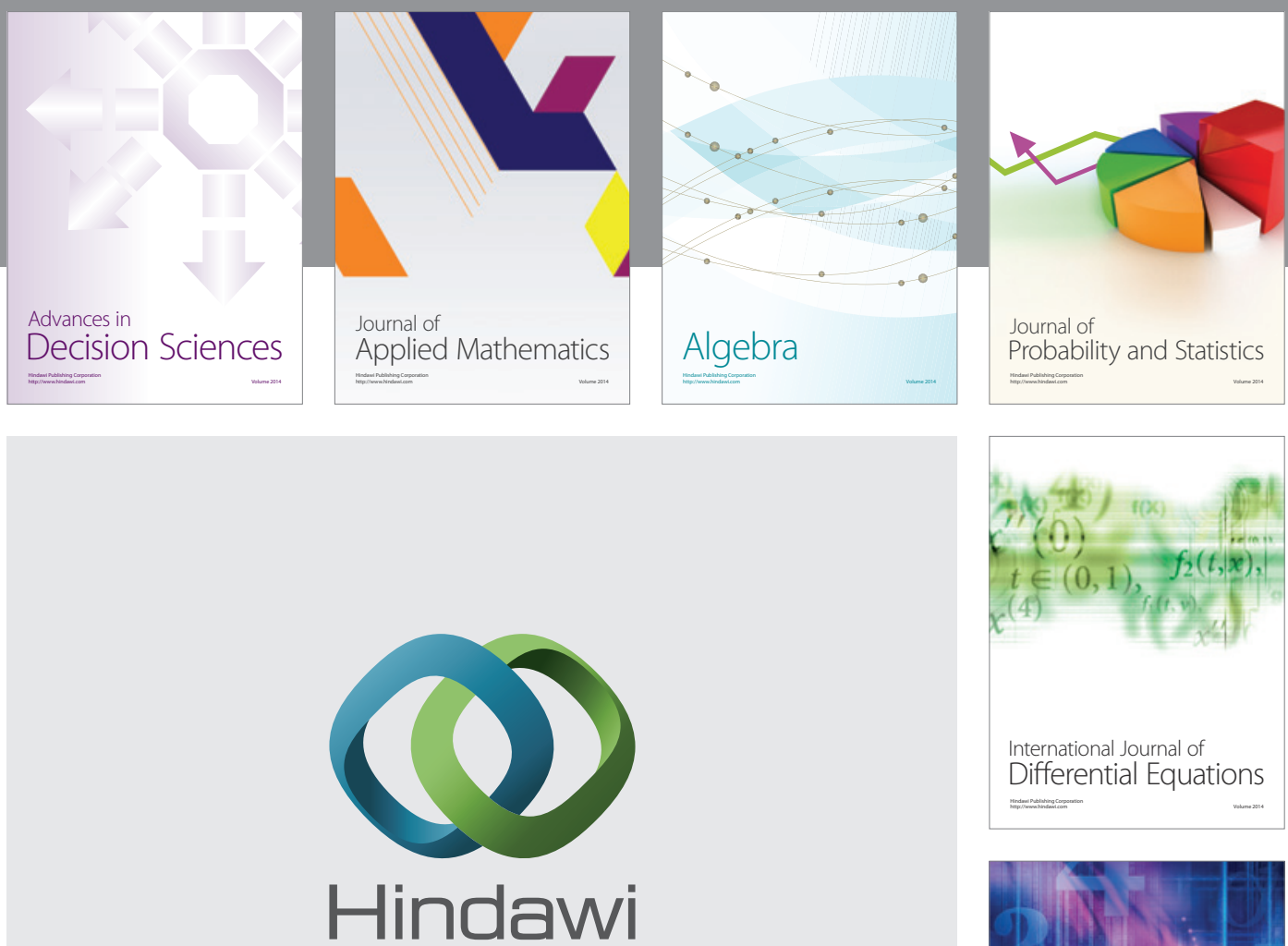

Submit your manuscripts at http://www.hindawi.com
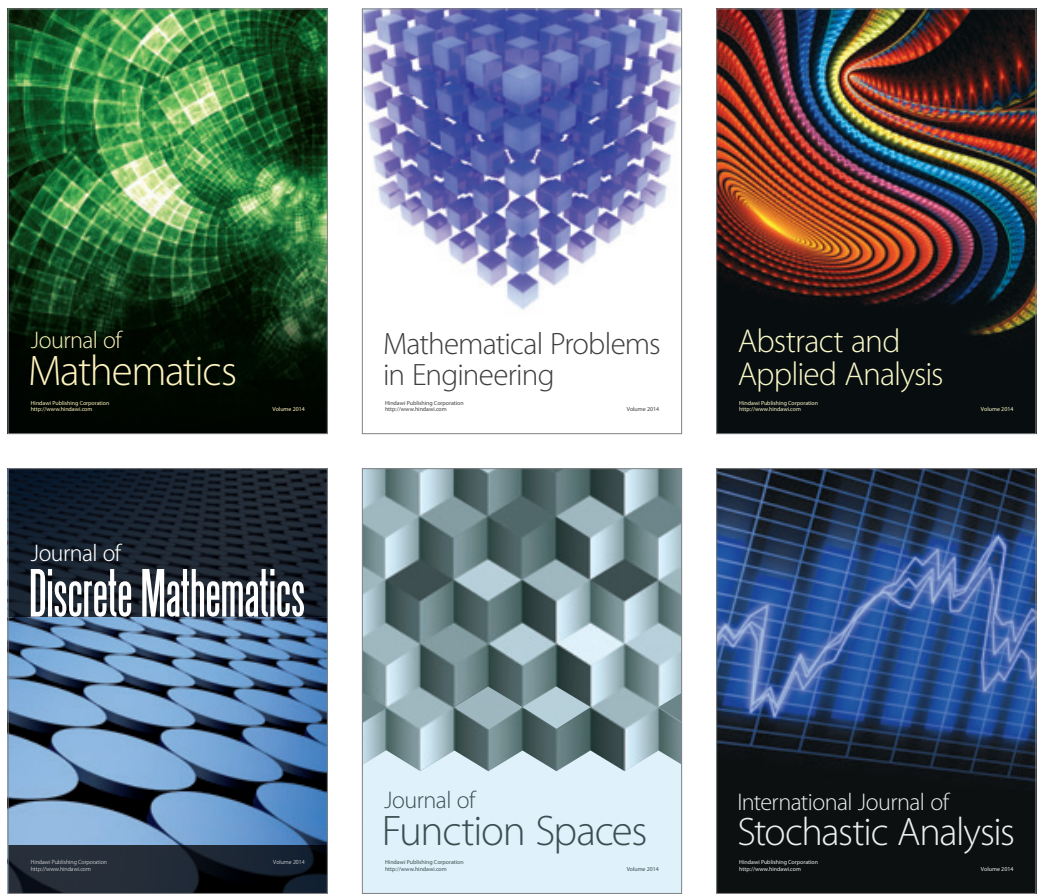

Journal of

Function Spaces

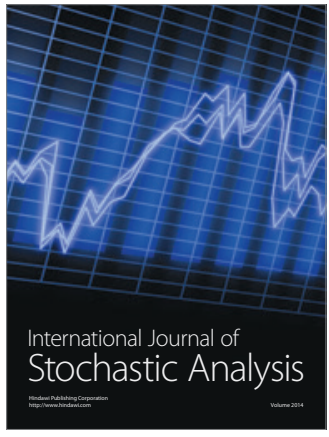

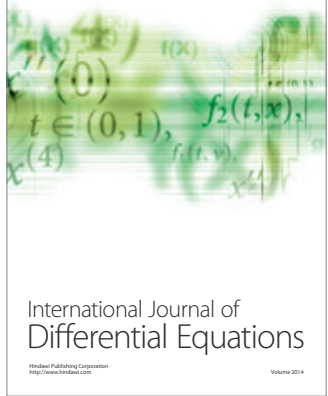
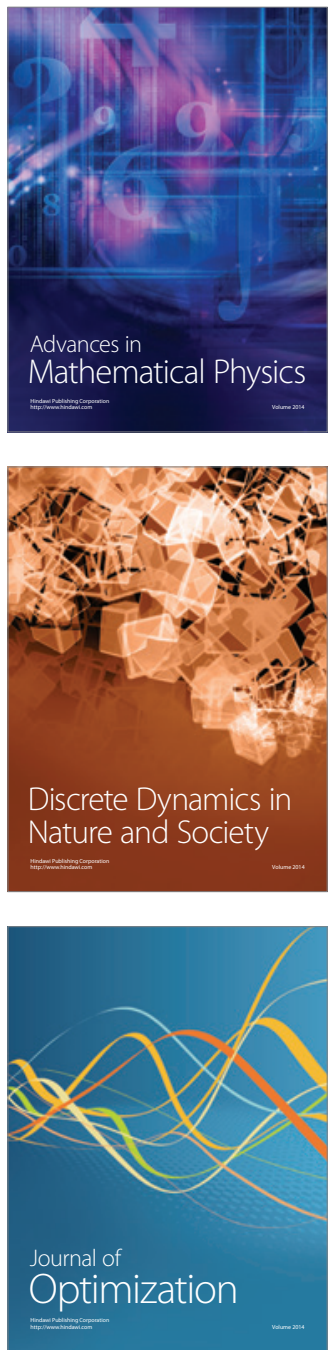